\title{
FORMA, FUNCIÓN Y SIGNIFICADO DE LA CONDUCCIÓN DE AUTOMÓVILES EN UN GRUPO DE ADULTOS MAYORES RESIDENTES EN SANTIAGO DE CHILE
}

\author{
FORM, FUNCTION AND MEANING OF CAR DRIVING IN A GROUP OF COMMUNITY- \\ DWELLING OLDER ADULTS IN SANTIAGO, CHILE
}

Jean Gajardo Jáuregui' . Diego Cifuentes Lucero²

\begin{abstract}
Resumen
Propósito: El objetivo de este trabajo fue indagar en características de la forma, función y significado de la conducción de automóviles en un grupo de adultos mayores residentes en la ciudad de Santiago de Chile, en el año 2011, a partir de sus experiencias y percepciones. Metodología: Bajo un paradigma naturalista interpretativo se realizó una entrevista en profundidad a siete participantes; luego la información fue analizada con un enfoque de teoría fundamentada por medio de comparación constante, y generando categorías con hallazgos/temas principales. Resultados: En el grupo estudiado, la conducción presentaba una forma condicionada por factores atribuibles al envejecimiento tales como declive visual, perceptual y cognitivo, y por el contexto físico y social. La función de la conducción emergió como medio facilitador de actividades instrumentales de la vida diaria y tiempo libre; y el significado de la conducción emergió como prioritario debido a su vinculación con la autonomía, la independencia y la continuidad biográfica al permitir la mantención de roles que eran importantes para los participantes. Conclusiones: Este trabajo posiciona la conducción de automóviles como una ocupación cuya forma, función y significado, contribuirían de manera importante al bienestar de los adultos mayores y releva información a considerar en los contextos de atención de Terapia Ocupacional para adultos mayores.
\end{abstract}

Palabras clave:

Vejez, conducción, ocupación, calidad de vida, autonomía.

\begin{abstract}
Purpose: The aim of this research was to inquire on characteristics of form, function and meaning of car driving in a group of community dwelling older adults in the city of Santiago de Chile, in 2011, from their perspectives and experiences. Methods: Qualitative in-depth interviews were carried out to seven participants; then information was analyzed using a grounded theory approach using constant comparison, and generating categories with main findings / themes. Results: Within the study group, car driving had a form conditioned by factors associable to aging such as visual, perceptual and cognitive declining, and by the social and physical context. Function of car driving emerged mainly as a facilitator of instrumental activities of daily living and leisure; and the meaning of the activity was probably the most important component, since car driving was perceived by the group as a priority and fundamental activity as it enabled independence, autonomy and biographical continuity by maintenance of significant roles. Conclusions: This study positions car driving as an occupation whose form, function and meaning, would contribute significantly to wellbeing and quality of life of older adults. Clinical implications for occupational therapy practice are also discussed.
\end{abstract}

Keywords

Aging, car driving, occupation, quality of life, autonomy.

1 Terapeuta Ocupacional, Licenciado en Ciencias de la Ocupación Humana, Máster en Gerontología Social. Profesor Asistente en Docencia, Escuela de Terapia Ocupacional. Facultad de Medicina. Universidad de Chile. Estudiante Doctorado en Salud Pública, Escuela de Salud Pública, Universidad de Chile. Becario Conicyt para estudios de doctorado en Chile. Independencia 1027. Tel 9786342.jgajardo@med.uchile.cl

2 Terapeuta Ocupacional, Licenciado en Ciencias de la Ocupación Humana, Diplomado en el Modelo de Ocupación Humana, Estudiante Máster Europeo en Terapia Ocupacional. Académico Escuela de Terapia Ocupacional. Facultad de Medicina. Universidad de Chile. Independencia 1027. Tel 9786589.dcifuentes@med.uchile.cl 


\section{INTRODUCCIÓN}

Conducir un automóvil es una de las actividades más complejas de la vida diaria en cualquier rango etario, ya que se desarrolla en un ambiente constantemente cambiante y desafiante como son las calles y el tráfico, e incorpora diversas habilidades cognitivas, funciones ejecutivas, motoras y perceptuales (Cutler, 2003). La investigación gerontológica en torno al transporte ha atribuido una importancia primordial al uso de auto privado como una estrategia de compensación de las restricciones de salud asociables con la edad (Scheiner, 2006).

Actualmente en Chile existen 67,1 personas de 60 años o más por cada 100 menores de 15 años, lo que se traduce en una proporción de $14,5 \%$ de personas de 60 o más años sobre la población total (Instituto Nacional de Estadísticas - INE, 2013). Se ha estimado que en el año 2050, esta población llegará a representar sobre un $25 \%$ de la población total del país (INE, 2002). Del mismo modo, la mayor parte de la población mayor reside en zonas urbanas (INE, 2013). A medida que la población mayor de 60 años aumenta, consecuentemente aumentará el número de conductores mayores.

Se ha previsto que se presentará un marcado aumento en el número de personas mayores conductoras de automóviles como resultado del envejecimiento poblacional y el aumento de la cantidad de automóviles de uso personal (US Transportation Research Board, 1988), es así como el automóvil es la forma más común de transporte usada por las personas mayores en Norteamérica (Kostyniuk \& Shope, 2003).

En el año 2005 se estimó que en Chile un 5,36\% del total de los adultos mayores chilenos conducía un automóvil como medio de transporte para realizar actividades diarias (Ramos \& Nazif, 2005). Considerando las proyecciones de otros países, el crecimiento del parque automotor y el acelerado envejecimiento de la población nacional, la conducción en la vejez emerge como un área de creciente interés, dados los cambios físicos, mentales y sociales que las personas experimentan al envejecer.

Desde una perspectiva biológica, la vejez se caracteriza por una reducción de la reserva funcional lo que incide en la capacidad de adaptación de las personas ante los desafíos diarios o el estrés cotidiano (Duarte \& Pedro, 2010). De este modo, existen diversas condiciones en la vejez que influyen en la forma de conducir y que pueden condicionar o determinar una experiencia exitosa al conducir el automóvil, entre éstas, podemos referir los cambios sensoriales, físicos y cognitivos, la presencia de condiciones de salud-enfermedad y el consumo de fármacos (Fernández-Ballesteros, 2000).

En un nivel internacional se ha seguido dos enfoques principales en el estudio de la relación de la vejez y la conducción: por un lado, existen los estudios epidemio lógicos en cuando a accidentes y por otra parte, existen trabajos experimentales sobre el deterioro que se produce con la edad en las habilidades y capacidades implicadas en la conducción (Romero y cols., 1990). No obstante, en Chile existe limitada información actual que permita un grado de conocimiento suficiente en ambas áreas.

La conducción de automóviles en la vejez ha sido también explorada con un énfasis por establecer el estado de adecuación de la persona mayor para llevar a cabo la actividad, o driving fitness, dado que en ocasiones las demandas propias de la conducción pueden superar las capacidades de los individuos, siendo probable que muchos conductores mayores auto-restrinjan o generen estrategias adaptativas en sus hábitos de conducción (limitando distancias, evitando conducir en la noche, conduciendo más despacio, evitando conducir con mal tiempo, evitando grandes vías, entre otros). Sin importar estos intentos por reducir y evitar el riesgo en la conducción, las tasas de accidentes de los conductores mayores se incrementan sutilmente desde los 60 años (Lewis, 2003; Klein, 1991). En Estados Unidos, por ejemplo, se conoce que las curvas de fatalidades en accidentes de tránsito según edad adquieren una distribución en " $u$ ", siendo los jóvenes y la tercera edad los más afectados (Zador, 1991).

Analizada como una ocupación que permite la movilidad en la comunidad, el uso de automóvil es una fuente importante de promoción de la independencia para las personas mayores y la restricción de esta actividad puede limitar seriamente el acceso a bienes y servicios, redes sociales y cuidado médico (Ostrow, Shafftron \& Pherson, 1992). Asimismo, el poseer una licencia de conductor y la posibilidad de conducir es de especial valor para la persona mayor ya que le permite mantener una identidad no relacionada con atributos de su edad ni con estigmatización (Eisenhandler, 1990). Así también, se ha identificado que las causas en el cese de la conducción de adultos mayores son frecuentemente económicas, médicas o relacionadas con pérdida de confianza, y lo que es más relevante, se ha logrado comprender que el cese de la conducción limita de manera relevante el estilo de vida (BishopMiller, Lewis \& Colledge, 2010). 
Desde una perspectiva de la ocupación, Nelson (1988) analiza la ocupación desde su forma, función y significado. La forma de la ocupación se refiere a los aspectos objetivos de la ocupación, aquello que es observable y que se manifiesta en la interacción actividadambiente, que ocurre en un tiempo y espacio determinados. La función de la ocupación, en cambio, tiene que ver con aquellos aspectos de la actividad que favorecen la adaptación al ambiente desde una perspectiva funcional, que se genera en la interacción personaactividad. Por último, el significado de la ocupación, son aquellos aspectos subjetivos, no observables directamente de la ocupación, que la persona construye en la interacción con el ambiente al realizar la actividad.

Dados los antecedentes señalados, por medio de este trabajo se buscó indagar en las características de la forma, función y significado de la actividad de conducción de automóviles de uso personal, en adultos mayores residentes en la ciudad de Santiago de Chile el año 2011, a partir de sus experiencias y percepciones en la actividad.

\section{Metodología}

Para responder al propósito de este trabajo la investigación se situó bajo un paradigma naturalista interpretativo con un enfoque fenomenológico, ya que éste se centra en la realidad en su contexto habitual, enfatizando en la subjetividad, significados e interpretaciones que las personas construyen a partir de sus experiencias (Goering \& Strauss, 1996).

A partir de las experiencias y percepciones de los participantes como conductores de automóviles de uso personal, se indagó en la forma, función y significado de la conducción de personas mayores chilenas residentes en Santiago. Desde esta dimensión de análisis, las metodologías cualitativas se vuelven una herramienta útil al momento de comprender el compromiso ocupacional en la conducción durante la vejez, ya que permite al investigador explorar el fenómeno de la ocupación, por una parte, desde aquellos aspectos más concretos, y por otra, indagar en la construcción de significados que la persona realiza a través de su compromiso en determinada actividad (Clarke, Wood \& Larson, 1998).

La información fue recolectada a través de la realización de una entrevista en profundidad a los participantes de la investigación.

La selección de participantes se realizó de manera deliberada, según los siguientes criterios de inclusión:

- Tener 60 años o más de edad,

- No tener diagnóstico de deterioro cognitivo, y

- Conducir a la fecha de la entrevista automóvil de uso privado (licencia tipo B).

En este trabajo participaron siete personas, cuatro mujeres y tres hombres, con un promedio de edad de 72,7 años, en un rango de 67 a 82 años. Todos los participantes poseían licencia de conductor tipo B y en el momento de la entrevista, todos conducían automóvil en su vida cotidiana. La tabla 1 muestra las características de los participantes.

Los participantes fueron contactados a partir de dos fuentes: el programa de voluntariado adulto mayor de la Municipalidad de Las Condes y la Facultad de Medicina de la Universidad de Chile. La figura 1 muestra el proceso de inclusión en la investigación. La tasa de reclutamiento fue de un $100 \%$, es decir, no se registrarón situaciones en las que los potenciales participantes desistieran de participar ya sea en el momento del contacto con el investigador como en el momento de la entrevista presencial. 
Tabla 1. Características de los participantes de la investigación

\begin{tabular}{|c|c|c|c|c|c|c|c|c|}
\hline Id & Sexo & Edad & $\begin{array}{l}\text { Estado } \\
\text { civil }\end{array}$ & $\begin{array}{l}\text { Nivel educativo } \\
\text { formal }\end{array}$ & Estado laboral & Comuna & $\begin{array}{c}\text { Años } \\
\text { conduciendo }\end{array}$ & $\begin{array}{l}\text { Antecedentes } \\
\text { médicos }\end{array}$ \\
\hline DdP & Hombre & 67 & Casado & $\begin{array}{c}\text { Profesional } \\
\text { universitario }\end{array}$ & $\begin{array}{l}\text { Jubilado, no } \\
\text { trabaja }\end{array}$ & $\begin{array}{c}\text { Las } \\
\text { Condes }\end{array}$ & Más de 45 & No \\
\hline GB & Hombre & 67 & Casado & $\begin{array}{l}\text { Profesional } \\
\text { universitario }\end{array}$ & $\begin{array}{l}\text { Jubilado, no } \\
\text { trabaja }\end{array}$ & $\begin{array}{l}\text { Las } \\
\text { Condes }\end{array}$ & Más de 40 & $\begin{array}{l}\text { Sd. Guillan Barré, déficit } \\
\text { motor en pierna izquier- } \\
\text { da, sobrepeso }\end{array}$ \\
\hline $\mathrm{AL}$ & Hombre & 78 & Casado & $\begin{array}{l}\text { Profesional univer- } \\
\text { sitario, Magíster }\end{array}$ & $\begin{array}{l}\text { Jubilado, no } \\
\text { trabaja }\end{array}$ & $\begin{array}{l}\text { Las } \\
\text { Condes }\end{array}$ & Más de 40 & No \\
\hline ET & Mujer & 73 & Viuda & $\begin{array}{l}\text { Profesional } \\
\text { universitaria }\end{array}$ & $\begin{array}{l}\text { Jubilada, no } \\
\text { trabaja }\end{array}$ & $\begin{array}{c}\text { Las } \\
\text { Condes }\end{array}$ & Más de 40 & $\begin{array}{c}\text { Cardiopatía, } \\
\text { Hipertensión arterial }\end{array}$ \\
\hline LV & Mujer & 82 & Casada & $\begin{array}{l}\text { Profesional univer- } \\
\text { sitaria, Magíster }\end{array}$ & $\begin{array}{l}\text { Jubilada, no } \\
\text { trabaja }\end{array}$ & $\begin{array}{c}\text { Las } \\
\text { Condes }\end{array}$ & Más de 55 & No \\
\hline RB & Mujer & 68 & Casada & $\begin{array}{l}\text { Profesional } \\
\text { universitaria }\end{array}$ & $\begin{array}{l}\text { Jubilada, } \\
\text { sigue } \\
\text { trabajando }\end{array}$ & $\begin{array}{l}\text { Las } \\
\text { Condes }\end{array}$ & Más de 30 & No \\
\hline SG & Mujer & 74 & Soltera & $\begin{array}{l}\text { Profesional uni- } \\
\text { versitaria }\end{array}$ & $\begin{array}{l}\text { Jubilada, } \\
\text { sigue traba- } \\
\text { jando }\end{array}$ & Ñuñoa & Más de 40 & Hipertensión arterial \\
\hline
\end{tabular}

Figura 1. Proceso de inclusión de participantes en la investigación

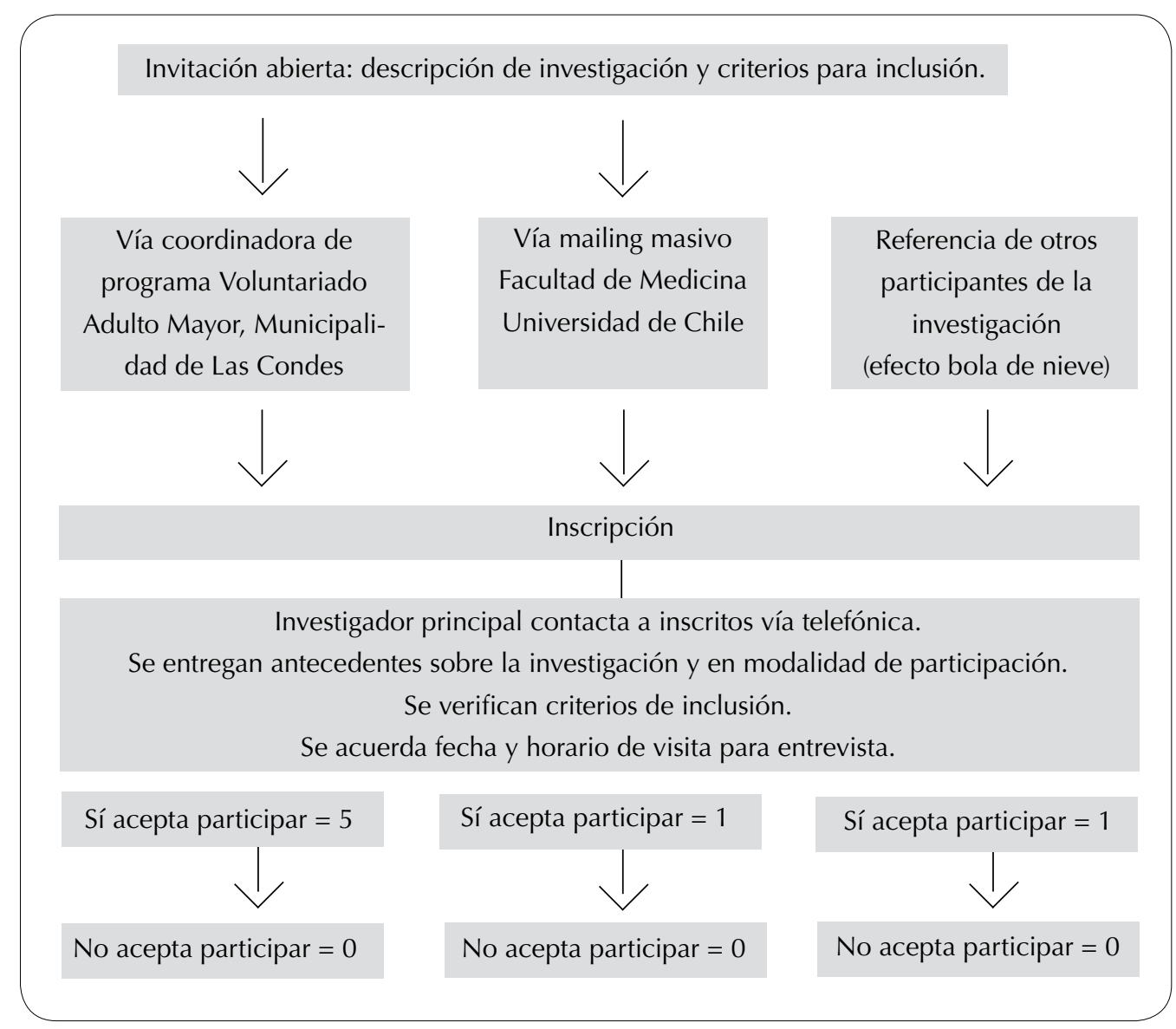


La recolección de la información se realizó por medio de una entrevista en profundidad a cada uno de los participantes. Las entrevistas fueron realizadas en un periodo de 2 meses entre marzo y abril 2011, e indagaron en diversos aspectos de la conducción en la vejez, a partir de las propias experiencias y percepciones de los participantes como conductores de automóviles en la ciudad de Santiago de Chile.

Las entrevistas se llevaron a cabo de forma individual, en los domicilios de los participantes, previo acuerdo de una fecha y hora de visita. Cada entrevista tuvo una duración de entre 30 a 60 minutos. Las entrevistas fueron audio-grabadas y posteriormente transcritas textualmente en programa Microsoft Word 1997-2003@.

Se seleccionó la técnica de entrevista en profundidad ya que permite riqueza informativa, es holística, es contextualizada, y es personalizada (Valles, 2003). Asimismo, la entrevista permitió acceder a información no prevista en el guión de entrevista, lo cual fue considerado por el equipo de investigación como un aporte dado el carácter exploratorio del trabajo de investigación y la insuficiente información que existe en Chile sobre la temática estudiada.

Se elaboró un guión de entrevista, con temas que se relacionasen con la forma, la función y el significado de la actividad de conducción. El guión fue utilizado como un respaldo para el entrevistador, dando prioridad durante la entrevista a la puntuación libre de temas por parte de la persona entrevistada. Algunas preguntas que formaron parte del guión de entrevista fueron:

¿Cómo adquirió su primer auto y por qué?

Como usted maneja ahora, ¿es diferente a como lo hacía en su adultez media o antes?

¿Para qué usa su automóvil hoy en día?

¿Cómo se siente hoy al manejar en relación con la seguridad de su conducción?

¿Cómo piensa que conducen las otras personas?

¿Es importante para usted conducir?

¿Qué pasaría si en su próximo control le niegan la licencia?

¿Cómo se sentiría si ya no pudiese manejar más?
El análisis de la información se realizó basado en un enfoque de teoría fundamentada por medio del método de comparación constante (Valles, 2003; La Cour, Josephsson \& Luborsky, 2005). El análisis inicial de las entrevistas se realizó escuchando cada entrevista por separado para favorecer la relación del investigador con su contenido. Con las entrevistas transcritas textualmente, se procedió a leer cada una por separado, y al mismo tiempo, iniciar la inclusión de códigos en los textos. Posteriormente, las unidades de texto codificadas fueron comparadas con otras con el mismo código, para proceder a vincularse en categorías iniciales; en esta etapa comenzaron a observarse temas emergentes, como los cambios en los hábitos de conducción asociados, la importancia de la actividad y la proyección negativa ante el futuro en el rol de conductor.

Luego, las categorías iniciales fueron comparadas entre sí y con las unidades textuales para continuar verificando su ajuste. Las categorías finales fueron elaboradas por medio del análisis comparativo de las categorías iniciales con antecedentes teóricos sobre aspectos gerontológicos implicados en la conducción y considerando su ajuste con el enfoque teórico fundamentado en la forma, función y significado de la ocupación.

\section{Consideraciones éticas}

La participación en este estudio no significó riesgo físico, psíquico ni moral para las personas implicadas. Todos los participantes firmaron consentimiento antes de ser entrevistados. Los propósitos del consentimiento informado fueron: verificar la comprensión de la información entregada en relación con los objetivos de la investigación y las características de su participación, explicitar la libertad de la participación, y autorizar al equipo de investigadores para usar la información obtenida con fines académicos y científicos resguardando la confidencialidad de los datos personales entregados.

Como parte del contenido de las entrevistas, no fueron encontradas eventuales conductas o actitudes narradas por los participantes que pudiesen requerir alguna instancia de consejería o educación con el fin de reducir riesgos vinculados al desarrollo de la actividad.

\section{Resultados}

Los resultados identificados luego del análisis de la información contenida en las entrevistas, representan 
hallazgos que permiten conocer con mayor profundidad aspectos de forma, función, y significado de la conducción de automóviles de adultos mayores chilenos.

De este análisis surgen las siguientes cuatro categorías de información que describen características de la conducción en el grupo de personas mayores.

\section{Forma de la conducción}

\section{Categoría 1: "Ahora ya no conduzco como lo hacía antes"}

Los participantes percibían que la vejez, en efecto, había cambiado su forma de conducir.

A partir de sus experiencias en la actividad de conducción, estos cambios que caracterizaban su forma de conducción en la vejez se ven reflejados en nuevas conductas y actitudes que antes no poseían, atribuyendo éstas a los cambios propios de la vejez que ellos percibían en su propia funcionalidad o en características de los contextos.

AL: "...uno se pone más prudente (al conducir), como más a la defensiva. Cuando uno es joven es más impetuoso. Ahora dejo más distancias. La experiencia que uno carga, pesa cuando uno es viejo..."

SG: "... antes andaba más rápido tratando de adelantar, lo que ahora no sucede porque tengo tiempo para hacerlo y además que no sé, creo que una se pone más generosa con la edad y maneja en forma más relajada...".

Los cambios en la forma de la conducción percibidos se relacionaban también con la percepción de variaciones de habilidades a nivel personal. Los participantes percibieron que el envejecimiento había traído consigo cambios en las diversas capacidades, y asociaron estos cambios con las habilidades necesarias para conducir. Los cambios que refirieron fueron en gran medida relacionados con las funciones cognitivas y sensoriales: disminución de la atención y de la velocidad de reacción, una memoria no tan eficiente como antes, reducción de la audición y la visión. Si bien reconocieron de amplia forma la presencia de cambios en estas áreas específicas, refirieron sentirse igualmente seguros al conducir que antes.
AL: "los reflejos no son los mismos, la audición tampoco... yo uso audífonos y hay una diferencia en el oído normal y el oído con audífono...".

LV: “...sé que la atención baja (en la vejez), no es la misma, la percepción de las distancias, sé que también eso cambia un poco. Uno no se percibe tan rápidamente del entorno. La atención es lo más importante...".

Del mismo modo, el contexto en el que se desarrollaba la actividad era percibido como influyente ya que los participantes refirieron que la ciudad de Santiago había sufrido cambios significativos en términos de arquitectura y tráfico de transporte, contexto que no era el que enfrentaban como conductores siendo más jóvenes, viéndose desafiados diariamente a nuevas experiencias que ellos percibían como restrictoras de la actividad.

GB: "...yo creo que efectivamente uno se va poniendo más lento en todas sus reacciones, eso es verdad, y por otra parte ve (cuando maneja) que el entorno es más agresivo... es cosa de ver, los adultos mayores andan en una velocidad menor, bueno, uno está expuesto ahí, hay que tomarlo con humor no más... Uno no se va a acelerar por unos garabatos, no...".

"...por otro lado se observa cómo el año pasado nunca se habían vendido tantos vehículos, doscientos y tantos mil... hay mucha gente que conduce muy desordenadamente.. Además yo creo que (hoy) se vive más aceleradamente en Santiago...".

\section{Función de la conducción}

\section{Categoría 2: "Mi auto me permite seguir haciendo lo que siempre me ha gustado hacer"}

La conducción permitía generar rutinas, mantener roles y vínculos sociales: Esta categoría ayuda a comprender la importancia que la actividad de conducción tiene para el grupo de personas mayores entrevistadas. Se observó que el automóvil permitía la realización de actividades diarias que tenían un significado para las personas, actividades que les permitían mantener una vinculación social con otras personas y mantener el control sobre sus roles, es por esto que la conducción de su automóvil era de alta importancia para el grupo. La conducción emergía como un facilitador del desa- 
rrollo de otras actividades instrumentales, como realizar compras, visitar a familiares y amigos.

DdP: "...Prácticamente lo uso a diario (el automóvil), ya sea para llevar a las personas que tengo a mi cargo en el voluntariado, ya sea para ir a buscar a mi señora a su trabajo o para ir a las compras de los súper o mercado o ir a la vega..."

LV: "Yo diría que (uso el automóvil) todos los días, ahora mi marido ya no tiene auto ni carné. Entonces necesariamente lo uso una vez al día para sacarlo a pasear, para hacer alguna gestión. También participo en un grupo social, y llego en auto al lugar y reuniones con el grupo directivo de este centro (del adulto mayor de Las Condes)...".

\section{Significado de la conducción}

\section{Categoría 3: "El auto me entrega libertad"}

La conducción del automóvil de uso personal era valorado como una herramienta que les permitía mantener su autonomía, enfatizando en la posibilidad de decidir cotidianamente en qué actividades involucrarse y con qué patrones de frecuencia. La conducción también era relacionada con un mejor estado de bienestar físico y afectivo.

SG: "... a mí me complica, me complica la vida pensar que no voy a ser capaz de manejar... trato de pensar positivamente... que está lejano... pero me sentiría mal, no creo que me deprima como para ir al psiquiatra...".

RB: "...creo que tendría que hacer una tremenda adaptación (si no pudiera maneja más), porque como resuelvo muchas cosas a través del auto... me cuesta imaginarlo... me sentiría más bien coartada en mi posibilidad de hacer más cosas... (el auto) me da libertad...".

\section{Categoría 4: "Mi futuro al volante es incierto y tiene fecha límite"}

Al analizar las entrevistas, surgió un punto relevante en relación con la forma en que los participantes proyectaban la actividad de conducción a medida que envejecieran. Las respuestas en este sentido tuvieron un contenido notoriamente homogéneo, denotando la percepción de incertidumbre en relación con su mantención en el futuro cercano. Los participantes refirieron que indudablemente van a dejar de conducir algún día, y relacionaron esta situación con factores de salud y con factores progresivos atribuibles al envejecimiento.

DdP: “... (dejaré de conducir) cuando sienta que ya no estoy capacitado, que me están fallando las reacciones, que me está fallando la audición, que me está fallando la movilidad, yo creo que ahí voy a dejar de manejar, porque no tengo por qué tengo que arriesgar la vida, ni la mía ni principalmente la de los demás...".

RB: "Sí, algún día va a tener que llegar eso (el cese de la conducción). Siento que por miles de razones, por vejez claro, también, quizás no voy a estar tan alerta, tendré algún accidente vascular...".

\section{Categoría 5: "Tengo que ocuparme de estar sano y así poder manejar".}

Dada la relevancia que la actividad de conducción tenía para los participantes, y ante la expectativa presente de máxima prolongación de la actividad, los participantes refirieron que habían incorporado otras actividades en su rutina diaria con el fin de favorecer un estado de bienestar que indirectamente potenciara el desempeño en la conducción misma y en las eventuales pruebas de conducción para renovación de licencia. De este modo, la actividad de conducción se había vuelto un activador de otras actividades en su rutina.

RB: "...espero mantenerme sana, por eso pinto, saco sudoku, hago yoga, trabajo la cabeza... lo hago con el objetivo de mantenerme bien...".

DdP: "...trato de cuidarme para que (la negación de licencia) llegue lo más tarde... sé que algún día, ya no me van a dar la licencia por edad, y ya no voy a manejar más...".

\section{DisCUSIÓN}

El objetivo de esta investigación fue indagar en aspectos de la forma, función y significado que adultos mayores chilenos asignan a la actividad de conducción 
de automóvil de uso personal. Los hallazgos obtenidos a partir de esta aproximación cualitativa entrega información novedosa en relación con dicho propósito.

Un primer aporte que puede mencionarse en relación con este trabajo, es que colabora en el desarrollo de información en un área en la que no existe mayor conocimiento a nivel nacional, la cual de la mano con el envejecimiento progresivo de nuestro país se vuelve más relevante. Del mismo modo, la consideración de un análisis desde la ocupación (a partir de la tríada forma, función, significado) permite establecer la atingencia de esta actividad para la Terapia Ocupacional, como una ocupación con características modificables y con aspectos de significado que la posicionan como relevante para las personas mayores.

Un primer hallazgo se relaciona con que, efectivamente, los participantes percibían que la forma que la conducción tenía en ellos había variado a la par con su envejecimiento individual. La actividad presentaba variaciones a partir de los cambios principalmente físicos y sensoriales atribuibles al envejecimiento. La importancia de los cambios individuales atribuibles al envejecimiento también eran relacionados por las personas en un nivel de creencias reflejado en las proyecciones ineludibles de cese de la actividad. Es notorio el énfasis que las personas participantes otorgaron a estos factores individuales como restrictores de la actividad, más que a otros factores extraindividuales (como el sistema actual de entrega de licencias). Los participantes refirieron también que el ritmo de vida acelerado y vertiginoso propio de una gran ciudad como Santiago, influía en el modo en que ellos afrontaban su conducción diaria.

Los adultos mayores participantes en este trabajo poseían educación formal de alto nivel, siendo todos profesionales universitarios y en algunos casos con estudios de posgrado. Del grupo, la mayor parte había jubilado de su actividad laboral y no había continuado realizando otras actividades productivas remuneradas. A partir de este estudio no es posible vislumbrar si dicho nivel socioeducativo podría considerarse como resultado de una muestra muy homogénea o si responde al perfil de la persona mayor que conduce automóvil de uso personal en Santiago de Chile.

Por medio del análisis de la función y el significado, fue posible notar que la conducción era una actividad relevante y hasta imprescindible en el grupo de participantes, ya fuese en aquéllos que mantenían una actividad laboral remunerada como en los que no, lo cual es concordante con lo propuesto por Marottoli y cols. (2000) en relación con el impacto del cese de la actividad en población estadounidense, y así también surgió como un elemento de continuidad biográfica, permitiendo mantener el desarrollo de actividades que solían desarrollar antes de jubilar. Este significado tendría coherencia desde la teoría gerontológica de la continuidad de Neugarten, la cual plantea que las personas buscamos a lo largo de nuestras vidas mantener la vigencia de las actividades y roles que son de importancia individual de acuerdo con las habilidades y el deseo de mantención de éstas (Covey, 1981).

La función del uso de automóvil se relacionó con actividades de diversas áreas: productivas remuneradas y no remuneradas (como un programa de voluntariado), tiempo libre, cuidado de otros (nietos, cónyuge), y actividades instrumentales de la vida diaria (realizar compras en supermercado, pagar cuentas y asistir al banco). Sobresale en este aspecto que el automóvil, más que un posibilitador de nuevas oportunidades, fue percibido como un mantenedor de las actividades que solía realizarse previamente, dependiendo de los roles existentes, es decir, en el grupo de personas mayores participantes la conducción favorecía su coherencia biográfica. Eisenhandler (1990) Ilama a esto mantención de una identidad individual no asociada a la edad ni a otros factores de estigmatización asociados con ésta.

Es fundamental resaltar la relevancia que tenía para el grupo el hecho de que la conducción facilitaba o permitía el desarrollo de actividades diarias que les ayudaban a mantenerse vinculados con otras personas y el mundo externo a su hogar. Este hallazgo es relevante si se considera, por ejemplo, que según la teoría de la desvinculación (Fernández-Ballesteros, 2000) se propone que en la persona mayor se produce una tendencia al cese de toda actividad que la ayude a integrarse socialmente. De este modo, en el grupo de participantes la conducción de automóviles podría considerarse un promovedor de vinculación.

Otro hallazgo relevante desde el significado de la conducción en los participantes se relacionó con el pesimismo implícito en su proyección del rol de conductor. Los participantes tenían la creencia que dejarían de conducir indudablemente algún día próximo, y que este cese de la actividad estaría relacionado con factores aparentemente asociados con la progresión de limitaciones sensoriales y físicas (velocidad de reacción, reflejos, visión, audición, fuerza y otros).

Este trabajo exploratorio permite visualizar áreas de investigación e intervención para la Terapia Ocupacional, con el propósito de aportar en la mantención de una ocupación que puede aportar al bienestar o que, 
por el contrario, puede poner en riesgo a una persona. Del mismo modo, abre reflexiones sobre, por ejemplo, la actual evaluación de conducción y el rol del Terapeuta Ocupacional en este proceso, desde una perspectiva de habilitación y rehabilitación. Por otra parte, emerge la relevancia de considerar a los grupos más vulnerables de conductores mayores, a partir de preguntas tipo: ¿cómo es la experiencia de las personas mayores que no logran renovar su licencia de conducir? ¿Cómo nuestro sistema les apoya en esta transición ocupacional, velando por la mantención de los roles y su autonomía? ¿De qué manera el sistema de transporte público se entrelaza para promover la inclusión social en estos grupos de personas?

\section{LIMITACIONES}

La entrevista se realizó una sola vez por participante. Si bien la repetición de la entrevista es una estrategia recomendada en algunas investigaciones con el objetivo de obtener mayor cantidad y profundidad de la información, la realización de sólo una entrevista por participante se consideró suficiente para el cumplimiento de los objetivos, ya que permitió acceder a la información deseada a un nivel exploratorio y se utilizó el principio de reiteración o saturación de información para determinar la suficiencia del número de entrevistas.

La transferibilidad de los hallazgos podría estar condicionada por las características sociodemográficas del grupo.

\section{AgradeCIMIENTOS}

Este estudio fue posible gracias a la participación de personas del programa de voluntariado Adulto Mayor de la Municipalidad de Las Condes y de académicos de la Facultad de Medicina de la Universidad de Chile, quienes nutrieron este trabajo con sus experiencias al volante.

El proyecto recibió apoyo económico por parte de la Fundación Universitaria Iberoamericana (FUNIBER).

\section{REFERENCIAS BIBLIOGRÁFICAS}

Bishop-Miller, F.; Lewis, S. \& Colledge, M. (1998). A community study of driving in the elderly. Age \& Ageing, 27(sup/ 1):46.

Clarke, F.; Wood, W. \& Larson, E. (1998). Occupational science: occupational therapy's legacy for the 21th century. En Blesedell, E.; Cohn, E.; Boyt, B. Willard \& Spackman: Terapia Ocupacional (Décima Edición). Madrid, España: Editorial Panamericana.

Covey, H. (1981). A Reconceptualization of Continuity Theory: Some Preliminary Thoughts. The Gerontologist, 21(6): 628-633.

Duarte, P.; Pedro, P. (2010). Terapia ocupacional en geriatría: principios y práctica. (Tercera edición). Barcelona: Editorial Elsevier Masson; 351-361.

Eisenhandler, SA. (1990). The asphalt identikit: Old age and the driver's license. International journal of aging and human development, 30(1):1-14.

Fernández-Ballesteros, R. (2009). Gerontología Social. Madrid, España: Editorial Pirámide.

Goering, P. \& Streiner, D. (1996). Reconcilable differences: the marriage of Qualitative and Quantitative Methods. Canadian Journal of Psychiatry, 41: 491-497.

Instituto Nacional de Estadísticas de Chile (2002). Índice general Censo 2002: Resultados y proyecciones. Recuperado el 5 de marzo de 2013 desde http://www.ine.cl/canales/usuarios/cedoc_online/censos/pdf/censo_2002_volumen_II.pdf

Instituto Nacional de Estadísticas de Chile (2013). Síntesis de resultados Censo 2012. Recuperado el 9 de abril de 2013 desde http:// www.censo.cl/contenido/sintesis_resultados_censo_2012.pdf

Klein, R. (1991). Age related disease, visual impairment and driving in the elderly. Journal of Human Factors,33: 521-5.

Kostyniuk, L. \& Shope, J. (2003). Driving and alternatives: older drivers in Michigan. Journal of Safety Resources, 34:407-14. La Cour, K.; Josephsson, S. \& Luborsky, M. (2005). Creating connections to life during life-threatening illness: Creative activity experienced by elderly people and occupational therapists. Scandinavian Journal of Occupational Therapy, 12: 98 - 109.

Lewis, S. (2003). Elder Care in Occupational Therapy. (Segunda edición). Estados Unidos: Slack incorporated.

Marottoli, R.; De León, C.; Glass, T.; Williams, C.; Coone, L. \& Berkman, L. (2000). Consequences of driving cessation: decreased out-of-home activity levels. Journal of Gerontology: Social Sciences, 55B: 334-340.

Nelson, D. (1988). Occupation: "form and performance". American Journal of Occupational Therapy, 42 (10): 633-41.

Ostrow, A.; Shafftron, P. \& Mc Pherson, K. (1992). The effects of a joint rage-of-motion physical fitness training program to improve automobile driving skills of older adults. Journal of Safety Resources, 23:207-19. 
Ramos, M.; Nazif, I. (2005). Adultos Mayores: Su interacción en el sistema de transporte terrestre. Instituto de Normalización Previsional de Chile. Recuperado desde http://www.conaset.cl/ cms_conaset/archivos/Estudio\%20conductual\%20adulto\%20 mayor.pdf

Romero, A.; García, J.; Martínez, F.; Morales, A. \& Sánchez, J. (1990). Conducción y vejez: el deterioro con la edad en tareas de velocidad de anticipación y coordinación visomotriz. Anales de Psicología, España, 6 (2): 221-231.

Scheiner, J. (2006). Does the car make elderly people happy and mobile? Settlement structures, car availability and leisure mobility of the elderly. European Journal of Transport and Infrastructure Research, 6(2): 151-172.

United States Transportation Research Board Council (1988). Transportation in an Ageing Society: improving mobility and safety for older persons. Special report 218. Recueprado el 10 de marzo de 2013 desde http://onlinepubs.trb.org/onlinepubs/conf/reports/cp_27.pdf

Valles, M. (2003). Técnicas cualitativas de investigación social: Reflexión metodológica y práctica profesional. (Tercera edición). Madrid, España: Editorial Síntesis.

Zador, P. (1991). Alcohol-related relative risk of fatal driver injuries in relation to driver age and sex. Journal of Studies on Alcohol, 52 (4): 302-10. 